\title{
Keeping an eye on the ring: COMS plaque loading optimization for improved dose conformity and homogeneity
}

\author{
Nolan L. Gagne, PhD', Daniel R. Cutright, PhD², Prof. Mark J. Rivard, PhD' \\ IDepartment of Radiation Oncology, Tufts University School of Medicine, Boston, MA, USA, 2Department of Radiation Oncology. \\ Northwestern Memorial Hospital, Chicago, IL, USA
}

\begin{abstract}
Purpose: To improve tumor dose conformity and homogeneity for COMS plaque brachytherapy by investigating the dosimetric effects of varying component source ring radionuclides and source strengths.

Material and methods: The MCNP5 Monte Carlo (MC) radiation transport code was used to simulate plaque heterogeneity-corrected dose distributions for individually-activated source rings of 14, 16 and 18 mm diameter COMS plaques, populated with ${ }^{103} \mathrm{Pd},{ }^{125} \mathrm{I}$ and ${ }^{131} \mathrm{Cs}$ sources. Ellipsoidal tumors were contoured for each plaque size and MATLAB programming was developed to generate tumor dose distributions for all possible ring weighting and radionuclide permutations for a given plaque size and source strength resolution, assuming a $75 \mathrm{~Gy}$ apical prescription dose. These dose distributions were analyzed for conformity and homogeneity and compared to reference dose distributions from uniformly-loaded 125I plaques. The most conformal and homogeneous dose distributions were reproduced within a reference eye environment to assess organ-at-risk (OAR) doses in the Pinnacle ${ }^{3}$ treatment planning system (TPS). The gamma-index analysis method was used to quantitatively compare MC and TPS-generated dose distributions.

Results: Concentrating $>97 \%$ of the total source strength in a single or pair of central ${ }^{103} \mathrm{Pd}$ seeds produced the most conformal dose distributions, with tumor basal doses a factor of 2-3 higher and OAR doses a factor of 2-3 lower than those of corresponding uniformly-loaded ${ }^{125}$ I plaques. Concentrating $82-86 \%$ of the total source strength in peripherally-loaded ${ }^{131} \mathrm{Cs}$ seeds produced the most homogeneous dose distributions, with tumor basal doses $17-25 \%$ lower and OAR doses typically $20 \%$ higher than those of corresponding uniformly-loaded ${ }^{125}$ I plaques. Gamma-index analysis found $>99 \%$ agreement between MC and TPS dose distributions.

Conclusions: A method was developed to select intra-plaque ring radionuclide compositions and source strengths to deliver more conformal and homogeneous tumor dose distributions than uniformly-loaded ${ }^{125}$ I plaques. This method may support coordinated investigations of an appropriate clinical target for eye plaque brachytherapy.
\end{abstract}

Key words: eye plaque, TG-43 hybrid technique, dosimetry, conformity index, homogeneity index.

\section{Purpose}

Investigations into the dosimetric and radiobiological effects of plaque size selection, radionuclide type, prescription dose and plaque positioning for eye plaque brachytherapy have been performed [1-5]. Recommendations exist regarding pre-operative quality assurance $(\mathrm{QA})$ procedures such as source strength verification using a well chamber with a NIST-traceable calibration [6,7]. Recent efforts have also been made to incorporate material and anatomical heterogeneities into treatment planning for eye plaque brachytherapy [7-11]. Yet despite these advancements, it remains primitive compared to other conventional brachytherapy modalities such as intracavitary accelerated partial breast irradiation and vaginal cylinder high dose-rate 192Ir brachytherapy [12], particularly with regards to conformity of the delivered dose to the clinical tumor volume. Additionally, though professional society recommendations are only available for COMS-plaques $[1,6]$, non-COMS eye plaque brachytherapy is practiced in many institutions worldwide [13-16]. Standardized and accurate dose calculation methods are required to facilitate evaluation of these dissimilar treatment devices.

The current prescriptive paradigm for eye plaque brachytherapy simply recommends that the prescription isodose line pass through the tumor apex and cover the entire tumor [1]. As no guidelines exist regarding tumor dose uniformity, typical COMS plaque tumor basal doses are

\footnotetext{
Address for correspondence: Mark J. Rivard, PhD, FAAPM, Department of Radiation Oncology Box \#246, Received: 07.05.2012 Tufts Medical Center, 800 Washington Street, Boston, MA 02111, USA, phone: +16176361680, 
a factor of 2.5-8 times higher than those at the apex [9]. For non-COMS plaques, tumor basal doses may be as much as 70 times higher than the prescription dose [17]. Such heterogeneous dose distributions risk toxicities in the proximal retina and sclera and inhibit identification of an angiogenic choroidal tumor base or a neoplastic advancing apical nevus as the true clinical target for eye plaque brachytherapy. Without knowledge of such a target, physical prescription dose de-escalation studies for eye plaque brachytherapy are unguided, and thus the same prescription dose has persisted for over the last 25 years, in contrast to other ocular melanoma treatment modalities such as stereotactic radiosurgery [18]. A dosimetric advantage of the COMS-plaques is the Silastic seed-carrier insert, which provides a reproducible source arrangement and dosimetric attenuation through tangential angles. To our knowledge, no research exists on the dosimetric effects of optimization methods such as varied intra-plaque source strengths and radionuclide choices or the clinical feasibilities therein for COMS-style eye plaque brachytherapy. The absence of such data restricts further coordinated refinement or dosimetric optimization of COMS-plaque eye brachytherapy towards achieving more conformal and uniform tumor dose distributions with less dose to critical structures. This study endeavors to explore these aspects.

\section{Material and methods}

\section{Rings}

The TG-43 hybrid technique [19] currently requires a cylindrically-symmetric dose distribution; this is due to

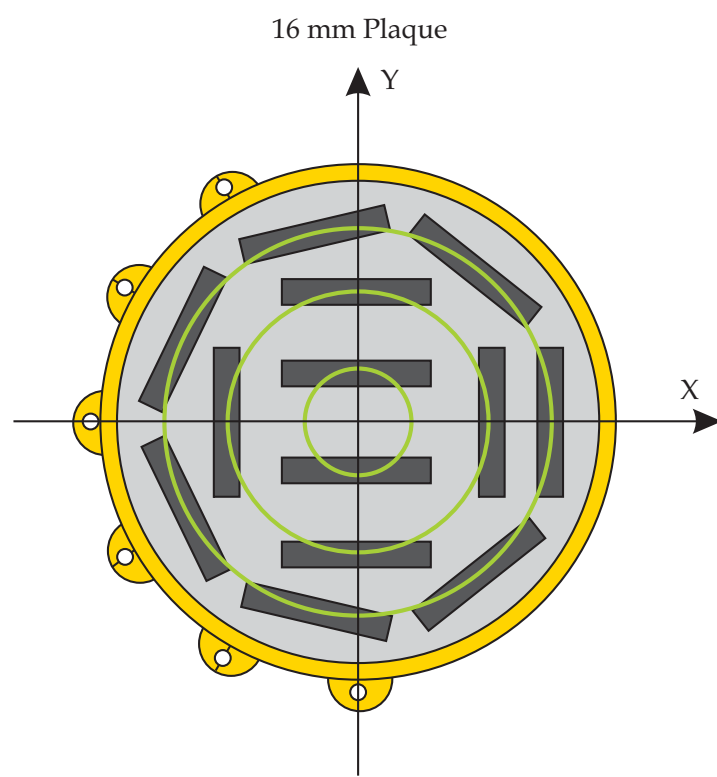

Fig. 1. Example of a $16 \mathrm{~mm}$ standard COMS-style eye plaque as viewed from the concave side of the Silastic insert (light grey). The plaque central-axis $\mathrm{Z}$ points out of the page (i.e., into the globe). The thirteen sources are designated by rectangles (dark grey), and the Modulay backing with six suture lugs (yellow) are also shown. Three constituent concentric rings of brachytherapy sources are shown (green). Figure adopted from Chiu-Tsao et al. [7]. treatment planning system (TPS) limitations with respect to TG-43 parameter [20] dataset entry. As such, it is outside the scope of this current investigation to explore the dosimetric effects of varying individual sources within a particular COMS-plaque. It is, however, possible to parse each COMS-plaque into constituent rings of sources, as the Silastic seed-carrier arranges sources about concentric circles of different radii (Fig. 1). In this manner, each COMS-plaque dose distribution may be considered as the superposition of these rings. The dosimetric influence of altering the source strength and radionuclide of component rings can then be explored. In this manuscript, the rings are enumerated in ascending order from the center of the plaque outward for reference: the $16 \mathrm{~mm}$ plaque in Fig. 1 thus consists of ring \#1 (innermost, 2 sources), ring \#2 (middle, 4 sources) and ring \#3 (outermost, 7 sources).

Following the methodology proposed above, the MCNP5 Monte Carlo (MC) radiation transport code [21] was used to simulate dose distributions for 14, 16 and $18 \mathrm{~mm}$ diameter COMS eye plaques with material compositions and reference geometry identical to those employed previously $[5,9]$. These plaque sizes were selected to investigate a range of customization capabilities afforded by their distinct numbers of rings (three in the $16 \mathrm{~mm}$ plaque, four in the $14 \mathrm{~mm}$ plaque and five in the $18 \mathrm{~mm}$ plaque). The plaques were populated with each of three distinct lowenergy brachytherapy sources: $103 \mathrm{Pd}$ (model 200, Theragenics, Corp. $\left.{ }^{\circledR}\right)$, 125I (model 6711, GE HealthCare, Inc. ${ }^{\circledR}$ ) and ${ }^{131} \mathrm{Cs}$ (model CS-1 Rev2, IsoRay Medical, Inc. ${ }^{\circledR}$ ). Source design specifications, absorbed dose determination methods and tally geometry were identical to those developed in Gagne et al. [5]. For the three radionuclides, each constituent ring of the three fully-loaded COMS plaques was activated to determine its contribution to the total dose distribution while accounting for the heterogeneous dosimetric influences of inter-seed attenuation and scatter. The dosimetric influence of replacing inactive seeds with air or water was also investigated for the range of plaques and radionuclides in this study to anticipate the possibility that the optimal COMS ring weighting for a particular tumor could involve the removal of one or more rings of seeds.

In a manner similar to Rivard et al. [19], a unique virtual source position and virtual set of brachytherapy dosimetry parameters was then ascribed to each ring. These brachytherapy dosimetry parameters were based on the AAPM TG-43 report formalism [20], and were formatted for Pinnacle 3 TPS (Philips Medical Systems, Cleveland, $\mathrm{OH}$ ) entry with a grid size of $0.2 \mathrm{~mm}$. Such virtual ring sources were used to investigate the dosimetric advantages of using distinct ring weightings with a single radionuclide or with intra-plaque radionuclide rings versus uniformly-loaded single-radionuclide COMS plaques.

\section{Ellipsoidal tumors}

Three clinically representative tumors (dome-shaped ellipsoids) with apical heights of 4,5 , and $6 \mathrm{~mm}$, basal diameters of 9, 11, and $13 \mathrm{~mm}$, and volumes of 144, 253, and $399 \mathrm{~mm}^{3}$ were contoured for the 14,16, and $18 \mathrm{~mm}$ COMS plaques, respectively. A MATLAB program (MathWorks, 
Natick, $\mathrm{MA}^{\circledR}$ ) was developed to generate tumor dose distributions for all possible ring weighting and radionuclide permutations for a given plaque size and source strength resolution. For example, with three radionuclides (103Pd, $125 \mathrm{I}$, and ${ }^{131} \mathrm{Cs}$ ) for each of the three rings of a $16 \mathrm{~mm}$ plaque, there are a total of $3^{3}=27$ possible combinations of radionuclide rings; this number scales exponentially with increased source strength resolution. Tumor dose distributions were generated in this study from $>10^{8}$ permutations of ring weightings and radionuclides for a given apical prescription dose. The total plaque heterogeneity-corrected physical prescription dose from all ring combinations for the three tumors was set to $75 \mathrm{~Gy}$ in agreement with the uniformly-loaded 125I plaque results of Melhus and Rivard [9] for equivalent prescription depths as considered in this study. This prescription dose is lower than the nominal $85 \mathrm{~Gy}$ - which assumes a homogeneous water calculation environment - to account primarily for the Modulay plaque, Silastic seed-guide insert, and interseed attenuation effects. ${ }^{125} \mathrm{I}$ was selected as the reference radionuclide for comparison due to its use in the COMS protocol.

\section{Unified dosimetry index analysis}

These ${ }_{T U M O R} D V H$ s were evaluated using a brachytherapy-specific adaptation of the unified dosimetry index (UDI) of Akpati et al. [22]. The UDI is originally defined as:

$U D I=\left\{\prod_{k=1}^{4} W_{k} \times\left\{\left|1-D I_{k}\right|+0.1\right\}\right\} \times 10^{4}$

The $D I_{K}$ are indices analyzing tumor coverage $\left(D I_{1}\right)$, dose conformity $\left(D I_{2}\right)$, dose homogeneity $\left(D I_{3}\right)$, and dose gradient $\left(D I_{4}\right)$. The UDI thus incorporates the weighted $\left(W_{k}\right)$ contributions from all four components into a single metric that scores the overall deviation of a treatment plan from an 'ideal' plan with UDI equal to unity.

Akpati et al. define $\mathrm{DI}_{3}$, the homogeneity index, as the ratio of the maximum dose at any point $2 \mathrm{~mm}$ beyond the PTV to the prescription dose. However, in COMS brachytherapy with the eye plaque sutured directly over the tumor, a more relevant investigation of tumor dose homogeneity would consider points most proximal to the plaque surface, such as at the tumor base. Though alternate homogeneity indices which incorporate volumetric considerations of the target have been investigated $[23,24]$, our definition of $\mathrm{DI}_{3}$ in this study preserved the point-dose-ratio nature of the original from Akpati et al. Moreover, since a fixed apical prescription point and appropriate tumor margin ensure excellent tumor coverage, the base-to-apex homogeneity of an eye plaque brachytherapy dose distribution is appropriately examined by investigating ${ }_{\text {TUMOR }} D_{M A X}$.

In an effort to improve clarity of the results and subsequent discussion, the symbolism employed in this study is slightly different than that of Akpati et al. As such, the individual $D I_{k}$ investigated were defined as:

$D I_{1}=$ Coverage Index $=\left\{\left|1-\frac{T U M O R}{T H 00 \%}\right|+0.1\right\} \times 10$,

$$
\begin{aligned}
& D I_{2}=\text { Conformity Index }=\left\{\left|1-\frac{V_{100 \%}}{\text { TUMOR V }}\right|+0.1\right\} \times 10, \\
& D I_{3}=\text { Homogeneity Index }=\left\{\left|1-\frac{T U M O R}{D_{R x}}\right|+0.1\right\} \times 10, \\
& \text { and } D_{4}=\text { Dose Gradient Index }=\left\{\left|1-\frac{V_{100 \%}}{V_{50 \%}}\right|+0.1\right\} \times 10,
\end{aligned}
$$

In Eqs. (2)-(5), TUMOR $V_{100 \%}$ is the tumor volume receiving $100 \%$ of the prescription dose (i.e., $75 \mathrm{~Gy}$ ), ${ }^{\mathrm{T} U M O R} V$ is the entire tumor volume, $V_{100 \%}$ is the entire eye volume receiving $75 \mathrm{~Gy}$ and, $V_{50 \%}$ is the entire eye volume receiving half of the prescription dose (i.e., 37.5 Gy). Volumes attributable to the plaque itself - the Modulay backing, Silastic insert, and radionuclide sources - were excluded from analysis entirely.

By their construction in the above equations, each $D I_{K}$ has an 'ideal' score of unity; one thus seeks to minimize these metrics in the treatment planning process. Equal weighting factors $W_{k}=1$ in Eq.(1) were assigned to the component metrics in this study. This prevented inflation/deflation of the absolute score, and as such the UDI was confined to a parameter space $\geq 1$ (see Fig. 1 from Akpati et al.), with an 'ideal' score of unity.

For the three tumor sizes, the component metrics $D I_{K}$ and overall UDI were calculated for each of the ${ }_{T U M O R} D V H \mathrm{~s}$, and compared to those of a uniformly-loaded (subscript "REF") ${ }^{125}$ I plaque. As will be discussed, the dose gradient of Akpati et al. (Eq. (5)) was found to be an inappropriate metric and was excluded from the overall UDI calculation. Thus, the overall UDI calculated for each candidate and reference plaque loading was determined by:

$U D I=\left\{\prod_{k=1}^{3} W_{k} \times\left\{\left|1-D I_{k}\right|+0.1\right\}\right\} \times 10^{3}$.

\section{TPS dosimetry}

To assess dosimetric effects to ocular organs-at-risk (OARs) versus uniformly-loaded 125I plaques, the three plaque loadings responsible for the most conformal $\left(D I_{2, M I N}\right)$, most homogeneous $\left(D I_{3, M I N}\right)$, and best overall (UDI MIN) tumor dose distributions for each plaque size were also simulated in a Pinnacle 3 TPS reference eye environment. This reference eye environment contained OAR contours for the ciliary body, cornea, eyelid, lacrimal gland, lens, and optic nerve, as well as dose points for the foveola and optic disc. Dose-volume histograms (DVHs) and maximum, minimum, and average doses from each plaque loading were calculated for these structures for comparison.

\section{Gamma-index analysis}

The method of Yang et al. [25] was employed to quantitatively compare the raw MC dose distribution data from a uniformly-loaded $16 \mathrm{~mm}$ plaque with the TPS dose distribution data from a summation of the plaque's three constituent rings using the OmniPro I'mRT software suite 
(IBA Dosimetry, Bartlett, TN). Both dose distributions were calculated with $(0.2 \mathrm{~mm})^{2}$ dose grid resolution, were normalized to $75 \mathrm{~Gy}$ at a depth of $5 \mathrm{~mm}$, and comprised a $(50 \mathrm{~mm})^{2}$ field-of-view.

\section{Results}

\section{Inactive seed locations}

The most dramatic dosimetric effects are observed when all but the active central ${ }^{131} \mathrm{Cs}$ seed of an $18 \mathrm{~mm}$ plaque are replaced with water (i.e., the assumption that all empty seed slots are filled with fluid upon implant). In this scenario, central axis doses are nonetheless only $<1.5 \%$ higher and off-axis doses only $<2.0 \%$ higher within the globe, with the maximum dose increase occurring near the scleral surface beneath the plaque edge. Assuming a $6 \mathrm{~mm}$ apical prescription depth of 75 Gy for this plaque, such a removal of radioactive sources leads to a total expansion of the $V_{50 \%}, V_{100 \%}$ and $V_{150 \%}$ isodose volumes of less than 24.0, 8.4, and $1.2 \mathrm{~mm}^{3}$, respectively. These effects were less pronounced for all other plaque sizes and radionuclide combinations considered. For instance, minimum central- and off-axis dose increases of $<0.6 \%$ within the globe resulted from an active central ${ }^{103} \mathrm{Pd}$ seed in a $14 \mathrm{~mm}$ plaque having all remaining slots filled with water.

\section{Ellipsoidal tumors}

\section{$D I_{1}$, coverage}

Since the 2003 ABS report [1] recommends that dose be prescribed to the tumor apex from an approximately cylindrically-symmetric arrangement of typically uniformlyloaded sources beneath and encompassing the base of the tumor, one might assume it is rare that the prescription isodose volume covers less than $95 \%$ of the tumor volume. However, a recent study has shown that following these recommendations, COMS eye plaque tumor coverage is a sensitive function of both prescription depth and assumed plaque placement uncertainty, and that $D_{95 \%}$ may be as low as $68 \%$ of $D_{R x}$ for certain lesions [26]. Nonetheless, the present study assumes that the plaque is placed perfectly coaxial above a cylindrically-symmetric tumor; likewise the tumor dimension and plaque size combinations in the present study also ensure adequate dose volumetric coverage. Thus, it is unlikely in the present study that any combination of radionuclide rings and source strengths would provide significantly better (or worse) coverage than a uniformly-loaded 125 I plaque with $D I_{1, R E F}=1.010,1.004$, and 1.000 for the $14 \mathrm{~mm}, 16 \mathrm{~mm}$, and $18 \mathrm{~mm}$ plaques, respectively.

Also, any combination of rings whose $75 \mathrm{~Gy}$ isodose volume at least encompasses the tumor scores a $D I_{1}$ of unity, and thus is indistinguishably 'better' than any other such combination with respect solely to this index. As such, further evaluation of candidate rings specifically with respect to coverage was abandoned in this study. The component $D I_{1}$ scores were nonetheless calculated and incorporated into the analysis of overall UDI for each ring combination as shown in Eq. (6).

\section{$\mathrm{DI}_{2}$, conformity}

For the $14 \mathrm{~mm}$ plaque, the most conformal tumor dose distribution (Fig. 2) had a $D I_{2, M I N}=6.2$ versus a $D I_{2, R E F}=$ 22.1 for the reference plaque, and was achieved by concentrating $>99 \%$ of the total source strength in the center $103 \mathrm{Pd}$ source. This improved conformity came at the expense of tumor dose homogeneity, resulting in a $\mathrm{DI}_{3}=50.4 \mathrm{vs}$. $D I_{3, R E F}=18.6$; ${ }_{\text {TUMOR }} D_{\text {MAX }}$ was also more than twice that of the reference $14 \mathrm{~mm}$ plaque.

For the $16 \mathrm{~mm}$ plaque, the most conformal tumor dose distribution (Fig. 2) had a $D I_{2, M I N}=5.8$ versus a $D I_{2, R E F}=$ 18.5 for the reference plaque, and was achieved by again concentrating $>99 \%$ of the total source strength in the innermost pair of $103 \mathrm{Pd}$ sources. This improved conformity again compromised tumor dose homogeneity, and resulted in a $D I_{3}=53.2$ vs. $D I_{3, R E F}=19.9 .{ }_{\text {TUMOR }} D_{M A X}$ was more than twice that of the reference $16 \mathrm{~mm}$ plaque.

For the $18 \mathrm{~mm}$ plaque, a minimum $D I_{2, M I N}=11.7$ (Fig. 2) was achieved versus a $D I_{2, R E F}=31.6$ by concentrating $>97 \%$ of the total source strength in one central ${ }^{103} \mathrm{Pd}$ source, with $<1 \%$ of the total source strength in the ${ }^{131}$ Cs sources filling each of rings \#2-\#5. This most conformal dose distribution resulted in a $D I_{3}=100.2$ vs. $D I_{3, R E F}=23.8$, and $\mathrm{a}_{\text {TUMOR }} D_{\text {MAX }}>800 \mathrm{~Gy}$, more than three times higher than that for a reference $18 \mathrm{~mm}$ plaque.

\section{$D I_{3}$, homogeneity}

The most homogeneous dose distribution for the $14 \mathrm{~mm}$ plaque (Fig. 2) had a $D I_{3, M I N}=11.8$ vs. $D I_{3, R E F}=18.6$. Rings $\# 1$ and \#2 were filled with $103 \mathrm{Pd}$ sources comprising $5.4 \%$ and $8.2 \%$ of the total source strength, respectively and rings \#3 and \#4 were filled with ${ }^{131}$ Cs sources accounting for the remaining $0.1 \%$ and $86.2 \%$, respectively. This resulted in a $23 \%$ reduction of ${ }_{T U M O R} D_{M A X}$ compared to a reference 125I plaque, albeit with compromised conformity: $D I_{2}=28.1$ as compared to $D I_{2, R E F}=22.1$.

For the $16 \mathrm{~mm}$ plaque, the most homogeneous dose distribution (Fig. 2) gave a $D I_{3, M I N}=15.1$ vs. $D I_{3, R E F}=19.9$. Ring \#1 was comprised of $103 \mathrm{Pd}$ sources having $15.2 \%$ of the total source strength and rings \#2 and \#3 filled with ${ }^{131} \mathrm{Cs}$ sources accounting for the remaining $0.1 \%$ and $84.7 \%$ of the total source strength, respectively. This resulted in a $17 \%$ reduction of ${ }_{T U M O R} D_{M A X}$ compared to a reference ${ }^{125} \mathrm{I}$ plaque, at the expense again of reduced conformity: $\mathrm{DI}_{2}$ $=21.0$ compared to $D I_{2, R E F}=18.5$.

For the $18 \mathrm{~mm}$ plaque, $D I_{3, M I N}=15.4$ vs. $D I_{3, R E F}=23.8$ was achieved (Fig. 2) with rings \#1, \#4, and \#5 filled with ${ }^{131}$ Cs sources of $0.7 \%, 0.9 \%$ and $82.2 \%$ of the total source strength, respectively. Rings \#2 and \#3 were filled with ${ }^{103} \mathrm{Pd}$ sources of $12.9 \%$ and $3.3 \%$ of the total source strength, respectively. This loading resulted in a $25 \%$ reduction of TUMOR $D_{\text {MAX }}$ compared to a reference ${ }^{125}$ I plaque, though conformity was compromised with $\mathrm{DI}_{2}=37.7$ compared to $D I_{2, R E F}=31.6$.

\section{$\mathrm{DI}_{4}$, dose gradient}

For the $14 \mathrm{~mm}$ plaque, $8 \times 10^{6}$ permutations of radionuclide and source strength could only achieve $D I_{4, M I N}=$ 6.22 vs. $D I_{4, R E F}=6.82$. The two other plaque sizes in this 


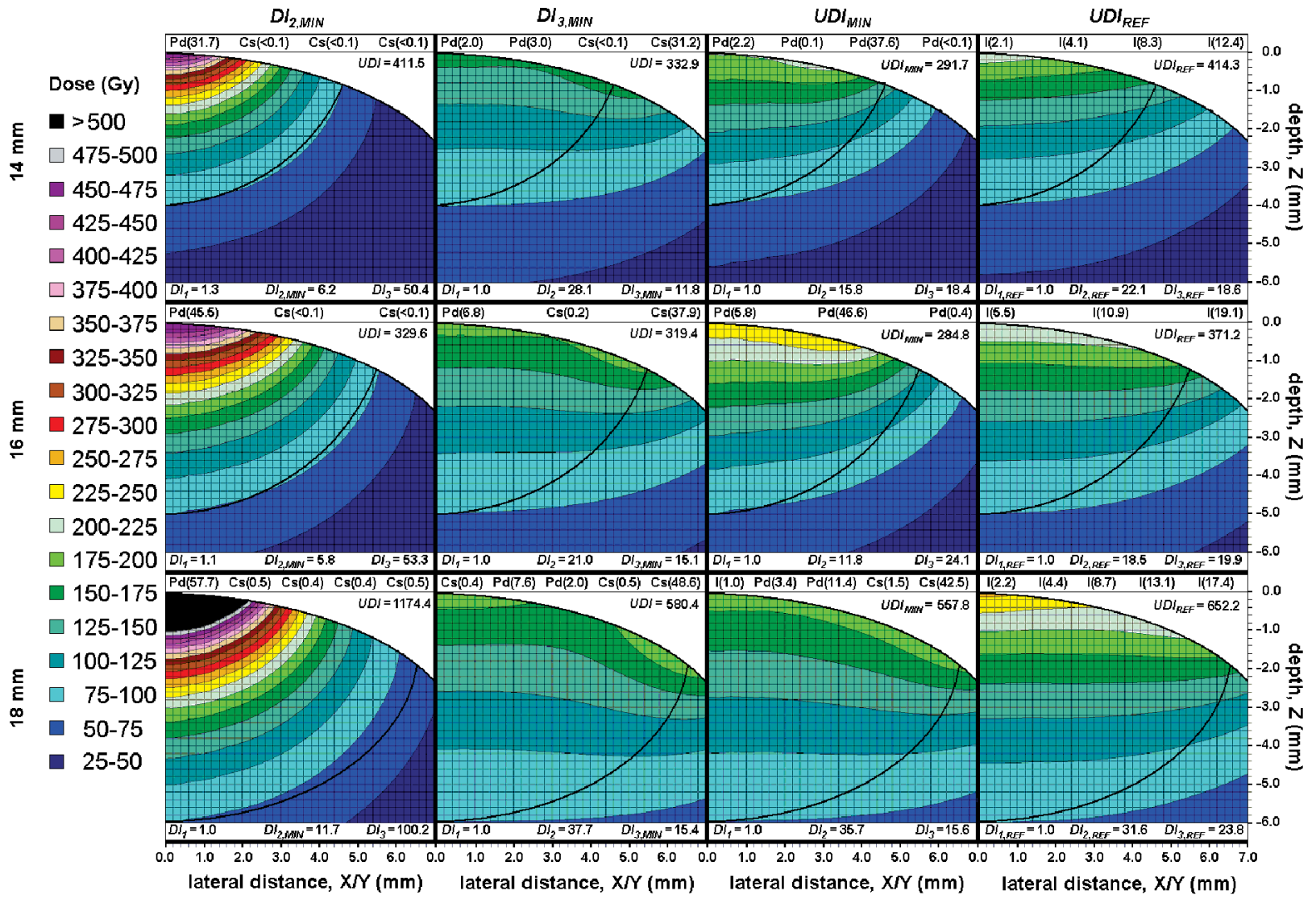

Fig. 2. Ellipsoidal tumor cross-sectional views of the most conformal $\left(D I_{2, M I N}\right)$, most homogeneous $\left(D I_{3, M I N}\right)$ and best overall $\left(U D I_{M I N}\right)$ dose distributions as compared to uniformly-loaded ${ }^{125} \mathrm{I}\left(U D I_{R E F}\right)$ for the $14 \mathrm{~mm}, 16 \mathrm{~mm}$ and $18 \mathrm{~mm}$ plaques. The radionuclide composition (" $\mathrm{Pd} "={ }^{103} \mathrm{Pd}$, "I" = ${ }^{125} \mathrm{I}$, “Cs" = ${ }^{131} \mathrm{Cs}$ ) and total source strength in $\mathrm{U}$ (parenthesized) of each constituent ring assuming a 7 day implant duration are provided at the top of the resultant dose distribution, with ring number increasing from left to right. The component metrics $\left(D I_{1}, D I_{2}\right.$, and $\left.D I_{3}\right)$ are provided at the bottom of each respective dose distribution, with the overall UDI score in bold at the upper-right corner of each figure. The ellipsoidal tumors are cylindrically-symmetric about $X / Y=0.0$. Tumor edges and the inner scleral edge are outlined in black. All volumes outside the inner sclera are in white at the upper-right corner of each figure

study demonstrated similar insensitivity of this metric to varied ring compositions and weightings. As discussed below, this index was found to be dosimetrically irrelevant for the purposes of the current study, and was excluded from calculation of the overall UDI results below.

\section{UDI}

Based on the $D I_{4}$ results above, the UDI in this study was a weighted product of $D I_{1}, D I_{2}$, and $D I_{3}$ only as expressed in Eq. (6). For the $14 \mathrm{~mm}{ }^{125} \mathrm{I}$ plaque, the lowest UDI (Fig. 2) was achieved using $103 \mathrm{Pd}$ in all four rings, with rings \#1-\#4 contributing $5.5 \%, 0.2 \%, 94.2 \%$ and $0.1 \%$ of the total source strength, respectively. This loading resulted in components $D I_{1}=1.01, D I_{2}=15.8$, and $D I_{3}=18.3$ for an overall $U D I_{M I N}=292 \mathrm{vs}$. $U D I_{R E F}=414$.

For the $16 \mathrm{~mm}$ plaque, the lowest UDI (Fig. 2) was achieved using $103 \mathrm{Pd}$ in all three rings with rings \#1-\#3 contributing $12.7 \%, 86.7 \%$ and $0.6 \%$ of the total source strength, respectively. This loading resulted in components $D I_{1}=1.01$, $D I_{2}=11.8$, and $D I_{3}=24.1$ for an overall $U D I_{M I N}=285 \mathrm{vs}$. $U D I_{R E F}=371$.
For the $18 \mathrm{~mm}$ plaque, the lowest UDI (Fig. 2) was achieved using ${ }^{125} \mathrm{I}$ in ring \#1, $103 \mathrm{Pd}$ in rings \#2 and \#3, and ${ }^{131} \mathrm{Cs}$ in rings $\# 4$ and \#5; the rings contributed $1.7 \%$, $5.7 \%, 19.1 \%, 2.4 \%$ and $71.1 \%$ of the total source strength, respectively. This loading resulted in components $D I_{1}=1.00$, $D I_{2}=35.7$, and $D I_{3}=15.6$ for an overall $U D I_{M I N}=558$ vs. $U D I_{R E F}=750$.

\section{TPS dosimetry}

Dose distributions in the reference eye environment for the $14 \mathrm{~mm}$ plaque loadings described above are shown in Figure 3 with resultant OAR DVHs shown in Figure 4. Table 1 provides OAR dose information for all three plaque sizes.

\section{Gamma-index analysis}

Employing the most stringent comparative criteria (i.e. a dose difference of $1 \%$, a distance difference of $1 \mathrm{~mm}$, a search distance of $1 \mathrm{~mm}$, and a $5 \%$ minimum relative signal) of the OmniPro I'mRT software, the gamma-index 


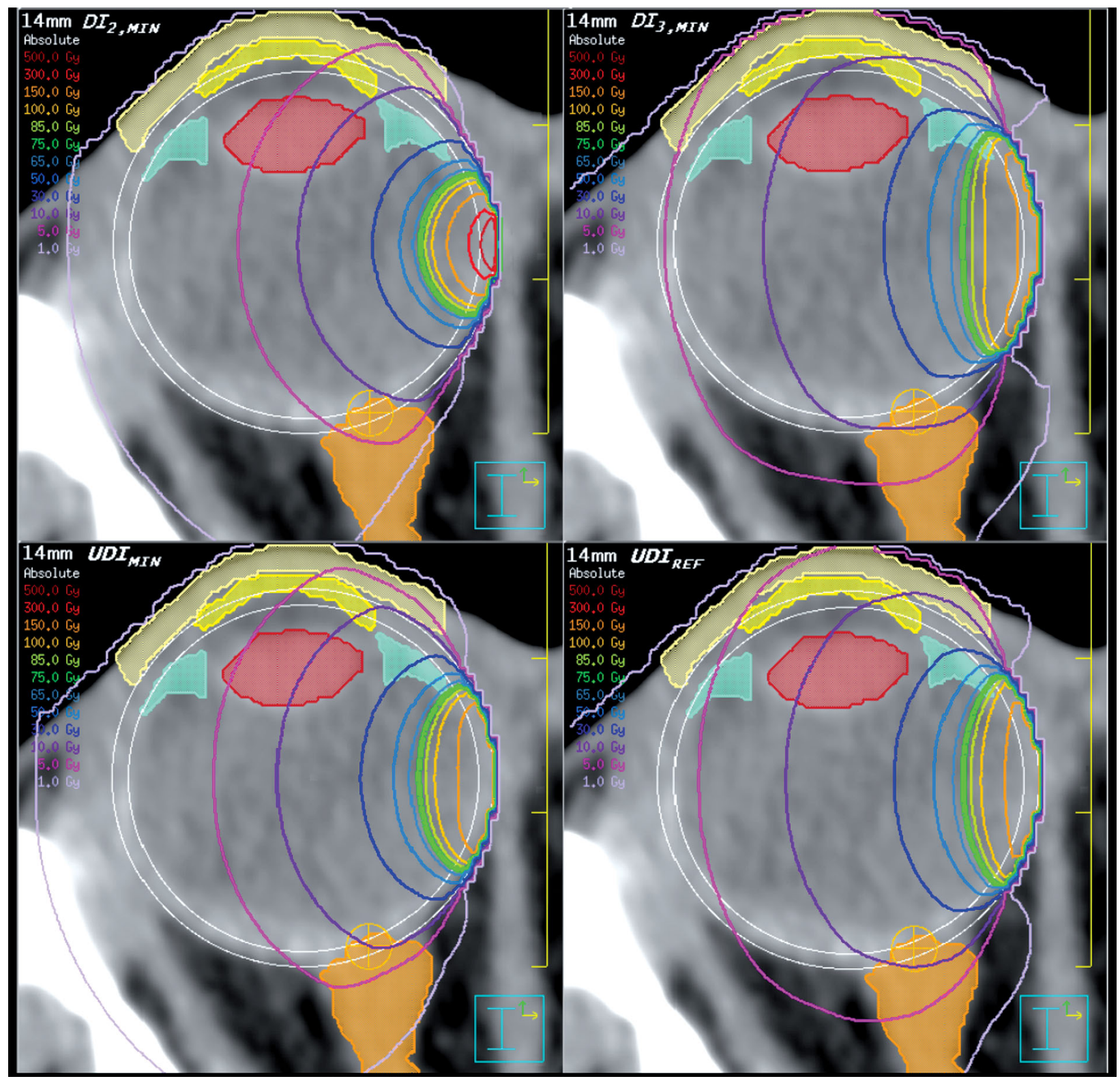

Fig. 3. Pinnacle 3 TPS dose distributions for the most conformal $\left(D I_{2, M I N}\right)$, most homogeneous $\left(D I_{3, M I N}\right)$, best overall $\left(U D I_{M I N}\right)$, and uniformly-loaded ${ }^{125} \mathrm{I}\left(U D I_{R E F}\right) 14 \mathrm{~mm}$ plaque loadings visualized on an axial (inferior/superior) cross section of a right (OD) eye at the nasal equator of the globe. OAR contours include the ciliary body (aqua), cornea (yellow), eyelid (beige), lens (red), and optic nerve (orange). Concentric circles establish the inner- and outer scleral boundaries (white), and a dose point simulates the optic disc (orange crosshair). The lacrimal gland and foveola are not pictured. Isodose lines are provided for 1, 5, $10,30,50,65,75,85,100,150,300$, and $500 \mathrm{~Gy}$, with the thick 75 Gy prescription isodose line in green

analysis of the full plaque MC and TPS rings dose distributions resulted in a $99.03 \%$ pass rate. These gamma-index results are shown in Figure 5.

\section{Discussion}

\section{Inactive seed locations}

Of the permutations of source ring removal investigated in this study, the most clinically relevant - based on the $D I_{2}$ results (Fig. 2 and Table 1 ) above - is perhaps the case of an activated center seed(s) with all other sources removed.
The case of a single central ${ }^{131} \mathrm{Cs}$ source in an $18 \mathrm{~mm}$ plaque somewhat unsurprisingly produces the largest of the observed dosimetric effects in this study. Firstly, this source position possesses the largest number of proximal neighbors, and is thus subject to the largest attenuation and scatter influences from the replacement of inactive surrounding high- $Z$ seeds with lower- $Z$ media such as air or water. Secondly, the higher energy $(\sim 30 \mathrm{keV})$ photon emissions from ${ }^{131}$ Cs likewise demonstrate the most pronounced lateral dosimetric effects as compared to the lower energy emissions from ${ }^{125} \mathrm{I}$ and ${ }^{103} \mathrm{Pd}$. Lastly, this scenario involves re- 

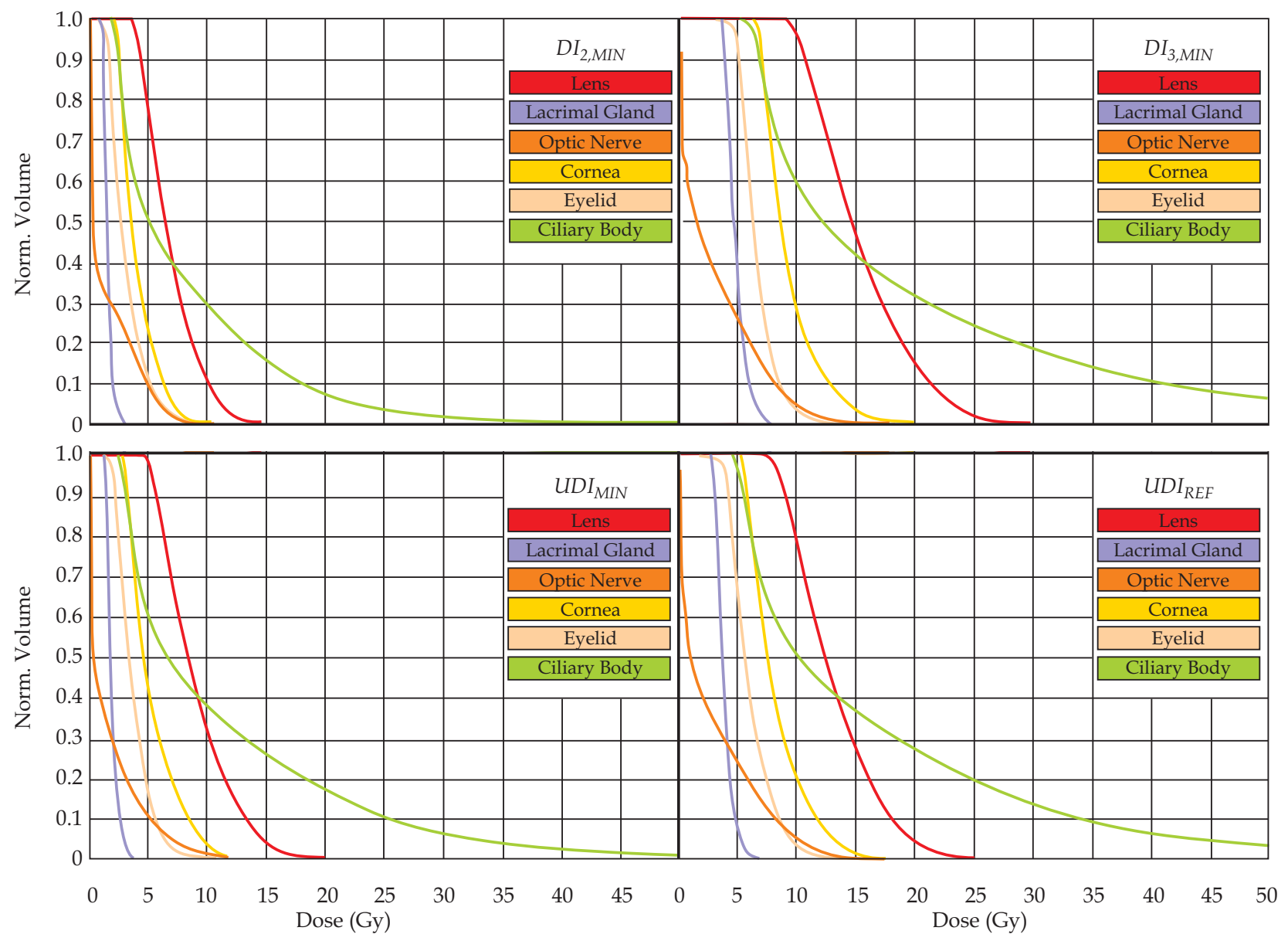

Fig. 4. OAR DVHs for the corresponding $14 \mathrm{~mm}$ plaque dose distributions from Figure 3

placement of $>95 \%$ (20 out of 21 ) of the plaque's seeds, whereas retention of any other radionuclide ring involves necessarily less substantial changes to the dosimetric environment (i.e., it intrinsically possesses more sources). Nonetheless, the results of this "worst case scenario" above indicate a negligible dosimetric effect due to the removal of inactive rings of seeds for the purpose of eye plaque brachytherapy dosimetric optimization. Similar dosimetric effects have been investigated previously and also deemed to constitute less than a $2 \%$ variation from a fully loaded plaque [27]. This is due in part to the source configurations of the COMS plaques having been designed explicitly to prevent interseed attenuation and shadowing and also attributed to the attenuating Silastic seed carrier insert.

\section{Ellipsoidal tumors}

\section{UDI calculations}

The UDI and component metrics of Akpati et al. were evaluated as applied to eye plaque brachytherapy in this study. As mentioned in the results above, tumor coverage (Eq. (1)) was not a significant concern given the assumptions of the current study, as well as the tumor dimensions and plaque sizes employed herein. As such, the optimization of this specific metric using the MATLAB code returned arbitrarily many candidate solutions whose dose distributions each resulted in the tumor volume being entirely encompassed by the 75 Gy isodose volume. For instance, all plaque loadings in Figure 2 provide nearly perfect coverage. These solutions are thus essentially equivalent with respect to coverage and are therefore of little interest to the reader or clinician in themselves. This metric was retained in the calculation of overall UDI, however, to emphasize the importance of tumor dose coverage while striving for better conformity or homogeneity.

Defined in Eq. (3) as the ratio of the entire eye volume (plaque excluded) receiving $75 \mathrm{~Gy}$ to the tumor volume, dose conformity for eye plaque brachytherapy is necessarily reduced by the presence of $1 \mathrm{~mm}$ thick sclera between the plaque and the tumor. Additionally, since plaque selection incorporates a 2-3 $\mathrm{mm}$ margin around the tumor base for conservative coverage [1], peripheral conformity to the tumor volume is largely absent in traditional uniformly-loaded plaques. Thus, the dosimetric flexibility provided by varied ring weightings and radionuclides resulted in the greatest improvement of this metric for all three ellipsoidal tumors. In this manner, $D I_{2}$ also became the most important component index in UDI calculations for an 'optimal' loading of the $14 \mathrm{~mm}$ and $16 \mathrm{~mm}$ plaques, assuming each $W_{k}=1$.

As shown in Figure 3 and Figure 4 for the $14 \mathrm{~mm}$ plaque, the most conformal plaque loadings resulted in the great- 
Table 1. OAR dosimetric information for the most conformal $\left(D I_{2}\right)$, most homogeneous $\left(D I_{3}\right)$, best overall $\left(U D I_{M I N}\right)$, and uniformly-loaded ${ }^{125}$ ( (UDI $\left.I_{R E F}\right) 14 \mathrm{~mm}, 16 \mathrm{~mm}$, and $18 \mathrm{~mm}$ plaque solutions positioned at the nasal equator of the globe, as shown in Figure 3. Minimum, mean, and maximum OAR doses are provided where appropriate and the reference plaque results are emboldened and italicized. Only point doses are provided for the foveola and optic disc, as these were simply dose calculation points rather than volumetric OARs. The '-' symbol for the eyelid and optic nerve indicate that these OARs had volumes outside the dose calculation grid which therefore received no physical dose. All values presented are in units Gy

\begin{tabular}{|c|c|c|c|c|c|c|c|c|c|c|c|c|c|}
\hline & \multicolumn{5}{|c|}{$14 \mathrm{~mm}$} & \multicolumn{4}{|c|}{$16 \mathrm{~mm}$} & \multicolumn{4}{|c|}{$18 \mathrm{~mm}$} \\
\hline & & $\mathrm{DI}_{2}$ & $\mathrm{DI}_{3}$ & $U D I_{M I N}$ & $U D I_{R E F}$ & $\mathrm{DI}_{2}$ & $\mathrm{Dl}_{3}$ & $U D I_{M I N}$ & $U D I_{R E F}$ & $\mathrm{DI}_{2}$ & $D I_{3}$ & $U D I_{M I N}$ & $U D I_{R E F}$ \\
\hline \multirow[t]{3}{*}{ Ciliary body } & $\min$ & 1.80 & 5.44 & 2.33 & 4.37 & 2.61 & 6.83 & 3.09 & 5.89 & 3.52 & 9.22 & 8.72 & 7.73 \\
\hline & mean & 8.05 & 19.11 & 11.40 & 16.15 & 11.87 & 25.49 & 15.44 & 22.82 & 15.59 & 37.02 & 35.35 & 31.42 \\
\hline & $\max$ & 59.23 & 145.11 & 106.13 & 123.10 & 92.05 & 175.99 & 146.18 & 167.14 & 113.22 & 191.93 & 188.67 & 195.91 \\
\hline \multirow[t]{3}{*}{ Cornea } & $\min$ & 2.12 & 6.22 & 2.78 & 5.05 & 3.08 & 7.86 & 3.70 & 6.85 & 4.15 & 10.68 & 10.10 & 9.02 \\
\hline & mean & 3.96 & 9.22 & 5.27 & 8.14 & 5.76 & 13.05 & 7.04 & 11.68 & 7.66 & 17.67 & 16.85 & 15.45 \\
\hline & $\max$ & 9.25 & 19.66 & 12.71 & 17.57 & 13.54 & 28.48 & 17.11 & 25.57 & 17.85 & 41.56 & 39.64 & 35.17 \\
\hline \multirow[t]{3}{*}{ Eyelid } & $\min$ & 0.45 & 1.90 & 0.74 & 1.54 & 0.54 & 1.82 & 0.67 & 1.53 & - & - & - & - \\
\hline & mean & 3.02 & 6.52 & 3.61 & 5.96 & 4.13 & 8.64 & 4.69 & 7.99 & 5.14 & 10.54 & 10.10 & 9.70 \\
\hline & $\max$ & 10.49 & 14.67 & 9.93 & 14.87 & 13.64 & 19.13 & 14.08 & 19.65 & 15.15 & 22.72 & 22.43 & 23.54 \\
\hline Foveola & & 3.74 & 9.55 & 4.94 & 8.09 & 5.44 & 12.47 & 6.60 & 11.06 & 7.25 & 17.16 & 16.32 & 14.68 \\
\hline \multirow[t]{3}{*}{ Lacrimal gland } & $\min$ & 0.94 & 3.42 & 1.22 & 2.66 & 1.36 & 4.30 & 1.62 & 3.59 & 1.87 & 5.79 & 5.43 & 4.71 \\
\hline & mean & 1.50 & 4.75 & 1.95 & 3.81 & 2.17 & 6.01 & 2.59 & 5.16 & 2.94 & 8.14 & 7.67 & 6.78 \\
\hline & $\max$ & 2.99 & 7.72 & 3.94 & 6.62 & 4.34 & 10.45 & 5.26 & 9.21 & 5.81 & 14.26 & 13.53 & 12.18 \\
\hline \multirow[t]{3}{*}{ Lens } & $\min$ & 3.42 & 8.85 & 4.47 & 7.32 & 4.96 & 11.14 & 5.94 & 9.90 & 6.64 & 15.12 & 14.40 & 13.02 \\
\hline & mean & 6.82 & 15.34 & 8.97 & 12.98 & 9.90 & 19.35 & 11.95 & 17.59 & 13.11 & 26.48 & 25.42 & 23.25 \\
\hline & $\max$ & 14.33 & 29.13 & 19.12 & 25.03 & 20.85 & 36.77 & 25.54 & 34.03 & 27.38 & 51.13 & 49.30 & 45.19 \\
\hline Optic disc & & 7.29 & 13.27 & 9.90 & 13.00 & 10.66 & 21.59 & 13.31 & 20.12 & 14.05 & 29.39 & 28.31 & 26.94 \\
\hline \multirow[t]{3}{*}{ Optic nerve } & $\min$ & - & - & - & - & - & - & - & - & - & - & - & - \\
\hline & mean & 1.42 & 2.91 & 1.61 & 2.69 & 1.87 & 3.72 & 2.06 & 3.47 & 2.16 & 4.33 & 4.15 & 3.97 \\
\hline & $\max$ & 9.71 & 18.24 & 13.45 & 17.25 & 14.25 & 29.87 & 18.17 & 27.24 & 18.80 & 44.71 & 42.63 & 38.11 \\
\hline
\end{tabular}
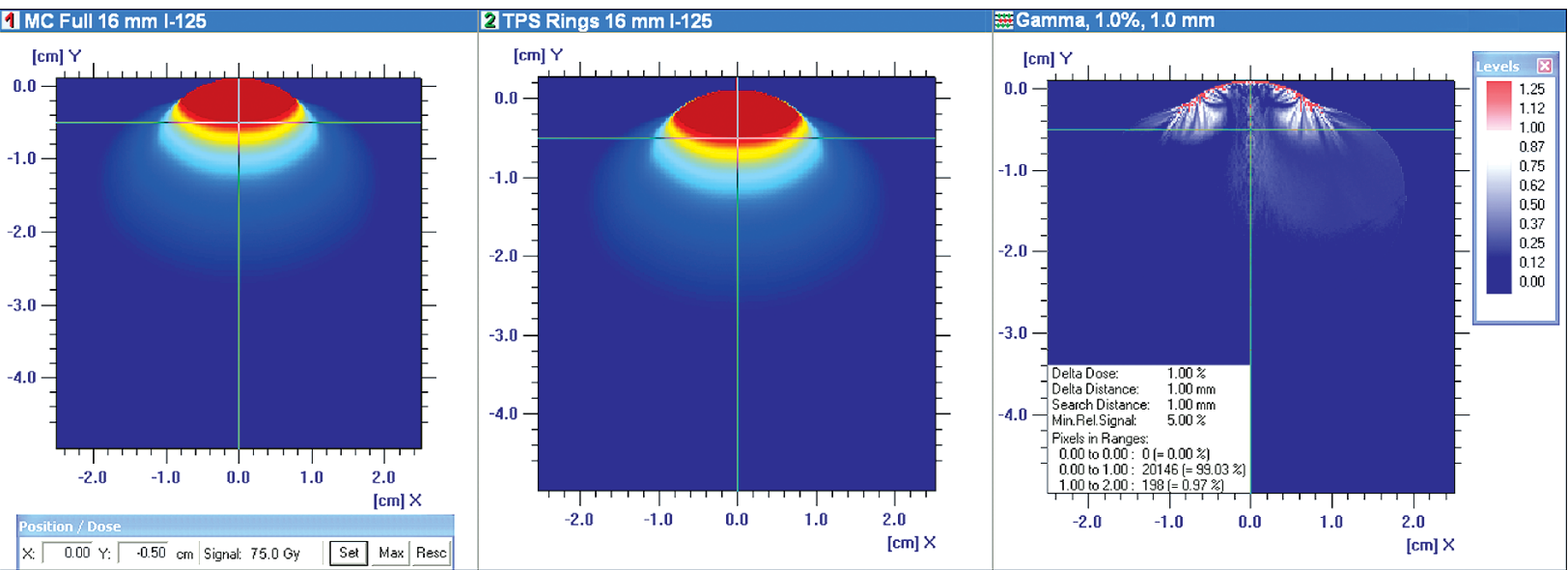

Fig. 5. Gamma-index analysis of the MC full ${ }^{125} \mathrm{I} 16 \mathrm{~mm}$ plaque dose distribution versus the TPS dose distribution from the sum of the three constituent $16 \mathrm{~mm}{ }^{125} \mathrm{I}$ plaque rings. Both dose distributions were normalized to $75 \mathrm{~Gy}$ at a depth of $5 \mathrm{~mm}$. Gamma-index results, parameters, and passing rate information are provided in the right-most image

est OAR sparing of those considered. Minimum, average, and maximum OAR doses were also typically 2 times lower for all plaque sizes than corresponding uniformly-load- ed ${ }^{125}$ I plaques (Table 1 ). This critical structure sparing, however, is offset by the $2 x-3 x$ increase in the tumor base dose versus that of the reference plaques, which likewise implies 
higher doses to the proximal sclera. These most conformal plaque loadings each employed a single central $103 \mathrm{Pd}$ seed (or pair of seeds for the $16 \mathrm{~mm}$ plaque) containing > 97\% of the total source strength to deliver the requisite dose distributions. This corresponded to source strengths of 23-57 U depending on plaque size. Such high-strength sources are beyond the current production capabilities of commercial brachytherapy source manufacturers, and also pose significant challenges to clinical implementation regarding health physics and quality assurance concerns [28].

Modification of the original $\mathrm{DI}_{3}$ from Akpati et al. in favor of that considered in this study - the ratio of the maximum and apical prescription tumor doses - nonetheless resulted in improved tumor dose homogeneity versus the reference plaques as shown in the second column of Figure 2. It is unlikely that more homogeneous tumor dose distributions could be produced using current COMS-style brachytherapy applicators given the limited range of photon energies for the three commercially-available radionuclides considered in this study, as well as the limited number of discrete source rings per plaque. This homogeneity came largely at the expense of conformity (Fig. 2) and OAR sparing (Figs. 3 and 4), however, due to the high strength ${ }^{131} \mathrm{Cs}$ seeds at the plaque peripheries. Though ${ }_{T U M O R} D_{M A X}$ was reduced by $17-25 \%$ depending on plaque size, the OAR doses in Table 1 were typically 1.2 times higher than those from the reference plaques.

As defined in Eq. (5), the dose gradient is a measure of the ratio of the prescription isodose volume, $V_{100 \%}$, to the half-prescription isodose volume, $V_{50 \%}$. For eye plaque brachytherapy, an ideal optimization of this index thus seeks to minimize the difference of these isodose volumes by shrinking the $V_{50 \%}$ more proximal to the tumor volume (sharper dose falloff), but the same mathematical effect is achieved by instead expanding the $V_{100 \%}$ to encompass more volume beyond the tumor while maintaining or decreasing the $V_{50 \%}$. The former is dosimetrically advantageous for eye plaque treatments and results in less dose to normal ocular structures adjacent to or opposite of the plaque, whereas the latter increases the volume of healthy eye receiving 75 Gy and risks increasing ocular toxicities. The three radionuclides in this study represent a relatively narrow range of average photon energies (20$30 \mathrm{keV}$ ) whose physical properties unfortunately prohibit significant reductions in $V_{50 \%}$ while maintaining a fixed apical prescription dose. The dose gradient of COMS eye plaque brachytherapy implants is thus somewhat limited in its improvement. The $\mathrm{DI}_{4}$ solutions in this study with expanded $V_{100 \%}$, however, point to the hazards of simple parameter optimization methodologies and underscore the importance of guided and thoughtful algorithmic programming. Applying a significantly lower weighting $W_{4}$ to this metric while increasing the $D I_{2}$ weighting $W_{2}$ may have constrained the MATLAB program to retain solutions with both improved conformity and steeper dose gradients, thus permitting its inclusion in the overall UDI calculations. The decision to maintain equal weightings $W_{K}$ in this study, however, was deliberate to emphasize the ambiguities surrounding selection of a clinically 'optimal' tumor dose distribution.
The best overall (i.e., $U D I_{M I N}$ ) plaque loadings for the $14 \mathrm{~mm}$ and $16 \mathrm{~mm}$ plaques produced more conformal dose distributions than the corresponding uniformlyloaded ${ }^{125}$ I plaques, with typically 1.6 times lower average OAR doses (Table 1). For the $18 \mathrm{~mm}$ plaque, however, the plaque loading resulting in the $U D I_{M I N}$ produced instead a more homogeneous tumor dose distribution than the reference loading, despite being less conformal. For this plaque size, the $\mathrm{DI}_{3}$ was the dominant component metric in the overall UDI calculations: with a $6 \mathrm{~mm}$ apical prescription depth, the conformity resulting from focusing source strength solely on inner plaque rings results in higher tumor base doses which quickly sabotage the $\mathrm{DI}_{3}$ score as seen in the first column of Figure 2. Thus, the optimal plaque loading positioned $71 \%$ of the total source strength in ${ }^{131} \mathrm{Cs}$ seeds in the outermost ring. Resultant OAR doses were typically $10 \%$ higher than those for the reference $18 \mathrm{~mm}$ plaque.

\section{Gamma-index analysis}

The gamma-index method for dosimetry quality analysis has been developed and widely used for IMRT patient QA to compare measured dose distributions with those generated from the treatment planning system. Application of this method directly to brachytherapy dosimetry is difficult since the clinical medical physicist does not measure brachytherapy dose distributions. Thus, an alternate application of the gamma-index method for brachytherapy dose distributions is to compare reference and planned results as investigated in Yang et al. [25]. The uniformly-loaded 125I plaque MC results represent the reference dose distributions in this study. The methods of this study parsed these full MC plaques into their individual rings for simulation, formatted virtual source parameter datasets from these $\mathrm{MC}$ ring results, entered these datasets into the TPS via the TG-43 hybrid technique, and created individual TPS ring dose distributions. By comparing these resultant TPS ring dose distributions against the standard fully-loaded plaque $\mathrm{MC}$ results, a quantitative dosimetric analysis of the entire process is permitted. The gamma-index results for the two $16 \mathrm{~mm}$ 125I dose distributions in Figure 5 demonstrate agreement within $1 \%$, and provide the dosimetric QA necessary for clinical adaptability of this technique.

\section{Implementation}

The candidate plaque loadings examined in this study offering improved conformity or homogeneity nonetheless present impediments to clinical implementation due to their employment of mixed radionuclides and non-uniform source strength weightings. Melhus and Rivard [9] provide plaque heterogeneity-corrected physical prescription doses for the range of COMS plaques yet assume the use of a single radionuclide per plaque. It is thus unclear what corrections should be applied to the ABS recommended homogeneous $85 \mathrm{~Gy}$ apical prescription dose for plaques containing a variety of radionuclide rings. Varied intraplaque radionuclide rings and source strength weightings also presents challenges to traditional clinical brachytherapy QA procedures. The importance of source distinction throughout the QA process from source strength verifica- 
tion to loading the Silastic insert could not be overstated in these instances.

As mentioned previously, the conformity requirements of a particular tumor dose distribution may lead to high concentrations of source strength at the upper tolerances of or even outside of current brachytherapy source manufacturer capabilities. Such high-strength sources may prove prohibitively expensive if not impossible to manufacture, and would present unique clinical concerns regarding personnel shielding and brachytherapy QA as well. Additionally, source manufacturers cannot always make seeds at the desired strength due to batching practices. This will quantize the available source strengths, especially for ${ }^{125} \mathrm{I}$, and limit the potential UDI $I_{\text {min }}$

Further, minimization of UDI $I_{\min }$ will be limited based on practical concerns such as potential for clinical medical physicists to measure low-energy source strengths to an accuracy $(k=2)$ of about $3 \%$.

\section{Limitations and future directions}

Though the dosimetric effects of inactive seed removal have been investigated and found negligible for the range of radionuclides and COMS plaques in this study, the plaque heterogeneity-corrected MC ring results presented herein assume that all Silastic seed slots in the plaque are filled with brachytherapy sources, active or otherwise. Additionally, these results are specific to the model $200(103 \mathrm{Pd}$, Theragenics Corp. ${ }^{\circledR}$ ), model 6711 (125I, Oncura/GE HealthCare Inc. ${ }^{\circledR}$ ) and model CS-1 Rev2 (131Cs, IsoRay Medical Inc. $\left.{ }^{\circledR}\right)$ sources. The relatively narrow range of lower photon energies offered by these commercially-available radionuclide sources also necessarily restricts the tumor dose homogeneity afforded by their utilization in an episcleral treatment methodology such COMS plaque brachytherapy.

The dosimetric customization demonstrated by the various plaque loadings in this study is necessarily limited by the fixed number of rings in the COMS plaque. Other plaque types with significantly greater numbers of sources [29] or surface patch-source modeling capabilities [30] likely provide improved customization. A further constraint in this investigation is the requirement that all brachytherapy sources within a given ring contain the same radionuclide. This limitation is due to the dosimetry parameter input requirements of current commercial TPS which confine the TG-43 hybrid technique [19] to cylindrically-symmetric calculation geometries. Further dosimetric customization is allowed by varying individual brachytherapy source strengths and radionuclides throughout the plaque, though these aspects are outside the scope of the current study.

The UDI of Akpati et al. was modified in this study to evaluate eye plaque brachytherapy dose distributions for coverage, conformity, and homogeneity. These dosimetric characteristics were considered with equal importance in the optimization process, though this method permits unequal weighting and thus custom target prioritization and OAR sparing on a per-patient basis. The $D I_{K}$ employed in these investigations are merely a sampling of the various quantitative metrics and evaluation criteria available to the clinical medical physicist, yet no such consensus objective measures currently exist towards identifying an 'op- timal' treatment specifically for eye plaque brachytherapy. Future research should investigate both the suitability and discriminatory ability of other evaluative metrics for this treatment modality.

\section{Conclusions}

A method has been developed for COMS plaque brachytherapy to select intra-plaque ring radionuclides and source strengths on an individual, per-patient basis to deliver more conformal and more homogeneous tumor dose distributions than a uniformly-loaded ${ }^{125}$ I plaque. The accurate, plaque heterogeneity-corrected dosimetry employed herein supports development of a prospective clinical protocol to determine the appropriate target (e.g., tumor base, tumor apex, tumor periphery, etc.) for eye plaque brachytherapy.

\section{References}

1. Nag S, Quivey JM, Earle JD et al. The American Brachytherapy Society recommendations for brachytherapy of uveal melanomas. Int I Radiat Oncol Biol Phys 2003; 56: 544-555.

2. Rivard MJ, Melhus CS, Sioshansi S et al. The impact of prescription depth, dose rate, plaque size, and source loading on the central axis using ${ }^{103} \mathrm{Pd},{ }^{125} \mathrm{I}$, and ${ }^{131} \mathrm{Cs}$. Brachytherapy 2008; 7: 327-335

3. Leonard KL, Gagne NL, Mignano JE et al. A 17-year retrospective study of institutional results for eye plaque brachytherapy of uveal melanoma using ${ }^{125} \mathrm{I},{ }^{103} \mathrm{Pd}$, and ${ }^{131} \mathrm{Cs}$ and review of the literature. Brachytherapy 2011; 10: 331-339.

4. Gagne NL, Leonard KL, Huber KE et al. BEDVH - a method for evaluating biologically effective dose volume histograms: Application to eye plaque brachytherapy implants. Med Phys 2012; 39: 976-983.

5. Gagne NL, Leonard KL, and Rivard MJ. Radiobiology for eye plaque brachytherapy and evaluation of radionuclide choice and implant duration using an objective function. Med Phys 2012; 39: 3332-3342

6. Butler WM, Bice Jr. WS, DeWerd LA et al. Third-party brachytherapy source calibrations and physicist responsibilities: Report of the AAPM Low Energy Brachytherapy Source Calibration Working Group. Med Phys 2008; 35: 3860-3865.

7. Chiu-Tsao S-T, Astrahan MA, Finger PT et al. Dosimetry of ${ }^{125} \mathrm{I}$ and ${ }^{103} \mathrm{Pd}$ COMS eye plaques for intraocular tumors: Report of Task Group 129 by the AAPM and ABS. Med Phys 2012; 39: 6161-6184.

8. Astrahan MA. Improved treatment planning for COMS eye plaques. Int I Radiat Oncol Biol Phys 2005; 61: 1227-1242.

9. Melhus CS, Rivard MJ. COMS eye plaque brachytherapy dosimetry simulations for ${ }^{103} \mathrm{Pd},{ }^{125} \mathrm{I}$, and ${ }^{131} \mathrm{Cs}$. Med Phys 2008; 35: 3364-3371.

10. Thomson RM and Rogers DWO. Monte Carlo dosimetry for ${ }^{125} \mathrm{I}$ and ${ }^{103} \mathrm{Pd}$ eye plaque brachytherapy with various seed models. Med Phys 2010; 37: 368-376.

11. Rivard MJ, Chiu-Tsao S-T, Finger PT et al. Comparison of dose calculation methods for brachytherapy of intraocular tumors. Med Phys 2011; 38: 306-316.

12. Rivard MJ, Venselaar JLM, and Beaulieu L. The evolution of brachytherapy treatment planning. Med Phys 2009; 36: 21362153.

13. Lommatzsch PK. Results after beta-irradiation (106 Ru/106Rh) of choroidal melanomas: 20 years' experience. Br J Ophthalmol 1986; 70: 844-885.

14. Granero D, Perez-Calatayud J, Ballester F et al. Dosimetric study of the $15 \mathrm{~mm}$ ROPES eye plaque. Med Phys 2004; 31: 3330-3336. 
15. Finger PT. Finger's "slotted" eye plaque for radiation therapy: treatment of juxtapapillary and circumpapillary intraocular tumours. Br J Ophthalmol 2007; 91: 891-894.

16. Thomson RM, Furutani KM, Pulido JS et al. Modified COMS plaques for ${ }^{125} \mathrm{I}$ and ${ }^{103} \mathrm{Pd}$ iris melanoma brachytherapy. Int J Radiat Oncol Biol Phys 2010; 78: 1261-1269.

17. Flühs D, Anastassiou G, Wening J et al. The design and the dosimetry of bi-nuclide radioactive ophthalmic applicators. Med Phys 2004; 31: 1481-1488.

18. Schirmer CM, Chan M, Mignano JE et al. Dose de-escalation with Gamma Knife radiosurgery in the treatment of choroidal melanoma. Int J Radiat Oncol Biol Phys 2009; 75: 170-176.

19. Rivard MJ, Melhus CS, Granero D et al. An approach to using conventional brachytherapy software for clinical treatment planning of complex, Monte Carlo-based brachytherapy dose distributions. Med Phys 2009; 36: 1968-1975.

20. Rivard MJ, Coursey BM, DeWerd LA et al. Update of AAPM Task Group No. 43 Report: A revised AAPM protocol for brachytherapy dose calculations. Med Phys 2004; 31: 633-674.

21. X-5 Monte Carlo Team. MCNP: a general Monte Carlo N-particle transport code, version 5. Los Alamos National Laboratory, Los Alamos 2003.

22. Akpati H, Kim C, Kim B et al. Unified dosimetry index (UDI): a figure of merit for ranking treatment plans. J Appl Clin Med Phys 2008; 9: 99-108.

23. Chaswal V, Yoo S, Thomadsen BR et al. Multi-species prostate implant treatment plans incorporating ${ }^{192} \mathrm{Ir}$ and ${ }^{125} \mathrm{I}$ using a Greedy Heuristic based 3D optimization algorithm. Med Phys 2007; 34: 436-444.

24. Yoon M, Park S, Shin D et al. A new homogeneity index based on statistical analysis of the dose volume histogram. J Appl Clin Med Phys 2007; 8: 9-17.

25. Yang Y, Melhus CS, Sioshansi S et al. Treatment planning of a skin-sparing conical breast brachytherapy applicator using conventional brachytherapy software. Med Phys 2011; 38: 15191525.

26. Gagne NL, Rivard MJ. Quantifying the dosimetric influences of COMS eye plaque radiation coverage and brachytherapy implant placement uncertainty on plaque size selection. Brachytherapy (2012-Ph16, accepted).

27. Thomson RM, Taylor REP, and Rogers DWO. Monte Carlo dosimetry for $125 \mathrm{I}$ and $103 \mathrm{Pd}$ eye plaque brachytherapy. Med Phys 2008; 35: 5530-5543.

28. Williamson JF, Dunscombe PB, Sharpe MB et al. Quality assurance needs for modern image-based radiotherapy: recommendations from 2007 interorganizational symposium on quality assurance of radiation therapy: challenges of advanced technology. Int J Radiat Oncol Biol Phys 2008; 71: S2-S12.

29. Astrahan MA, Luxton G, Pu Q et al. Conformal episcleral plaque therapy. Int J Radiat Oncol Biol Phys 1997; 39: 505-519.

30. Astrahan MA. A patch source model for treatment planning of ruthenium ophthalmic applicators. Med Phys 2004; 30: 12191228 . 